\title{
DOC sources and DOC transport pathways in a small headwater catch- ment as revealed by carbon isotope fluctuation during storm events
}

T. Lambert et al.

Correspondence to: T. Lambert (tlambert@ulg.ac.be) 
2 Supplementary Table 1: Discharge, DOC concentration and isotopic composition and major anion

3 concentrations for stream waters sampled at the outlet of the catchment during the six storm events $4 \quad$ (n.d. = no data).

5 Supplementary Table 2: Carbon isotopic composition of SOC, Water Extractable Organic Carbon 6 (WEOC) and DOC in the uppermost $(0-15 \mathrm{~cm})$ soil horizons in riparian wetland and upland 7 domains. WEOC was obtained from air-dried soil samples mixed with deionized water (soil:water 8 ratio $=1 / 4)$, shaken during 4 hours at $250 \mathrm{rpm}$ then filtered at 0.7 and then $0.2 \mu \mathrm{m}$ (n.d. $=$ no data).

9 Supplementary Figure 1: Temporal variations in nitrate and sulfate concentrations at the catchment outlet during the study period. Monitored storm events are indicated by numbers. 
Supplemental Table 1

\begin{tabular}{|c|c|c|c|c|c|c|c|}
\hline Event & Date & Discharge $\left(L s^{-1}\right)$ & DOC $\left(\mathrm{mg} \mathrm{L}^{-1}\right)$ & $\delta^{13} \mathbf{C}_{\text {DOC }}(\% o)$ & $\mathrm{Cl}\left(\mathrm{mg} \mathrm{L}^{-1}\right)$ & $\mathrm{NO}_{3}\left(\mathrm{mg} \mathrm{L}^{-1}\right)$ & $\mathrm{SO}_{4}\left(\mathrm{mg} \mathrm{L}^{-1}\right)$ \\
\hline \multirow[t]{22}{*}{ Event 1} & $11 / 11 / 104: 00$ & 18.3 & 11.5 & -28.1 & 35.9 & 50.8 & 18.6 \\
\hline & $11 / 11 / 1010: 27$ & 28.8 & 13.2 & -28.6 & 33.1 & 39.2 & 16.0 \\
\hline & 11/11/10 11:07 & 46.0 & 13.5 & -28.5 & 32.8 & 43.4 & 15.5 \\
\hline & 11/11/10 11:47 & 63.3 & 13.5 & -28.4 & 32.1 & 40.8 & 15.2 \\
\hline & $11 / 11 / 1012: 27$ & 78.8 & 14.1 & -28.3 & 31.1 & 38.0 & 14.9 \\
\hline & 11/11/10 13:07 & 89.9 & 15.8 & -28.4 & 30.2 & 35.9 & 15.1 \\
\hline & $11 / 11 / 1013: 47$ & 90.9 & 16.7 & -28.5 & 30.5 & 35.1 & 15.0 \\
\hline & $11 / 11 / 1014: 27$ & 90.9 & 17.4 & -28.3 & 30.6 & 35.7 & 15.2 \\
\hline & $11 / 11 / 1015: 07$ & 90.9 & 17.5 & -28.3 & 31.1 & 36.8 & 15.6 \\
\hline & $11 / 11 / 1015: 47$ & 88.8 & 17.6 & -28.4 & 31.9 & 38.4 & 16.3 \\
\hline & $11 / 11 / 1016: 27$ & 85.8 & 17.8 & -28.3 & 32.5 & 40.1 & 17.2 \\
\hline & 11/11/10 17:07 & 80.8 & 17.6 & -28.0 & 33.0 & 41.1 & 17.5 \\
\hline & $11 / 11 / 1017: 47$ & 76.8 & 17.3 & -28.2 & 32.8 & 42.6 & 17.7 \\
\hline & $11 / 11 / 1018: 27$ & 71.9 & 17.1 & -28.2 & 33.4 & 44.3 & 17.8 \\
\hline & 11/11/10 19:07 & 69.1 & 16.7 & -28.1 & 34.1 & 45.5 & 18.6 \\
\hline & $11 / 11 / 1019: 47$ & 66.2 & 16.5 & -27.8 & 33.8 & 46.0 & 18.6 \\
\hline & 11/11/10 21:07 & 64.3 & 16.6 & -27.9 & 34.9 & 49.3 & 19.1 \\
\hline & $11 / 11 / 1022: 27$ & 63.3 & 16.2 & -27.8 & 34.4 & 49.3 & 18.7 \\
\hline & $11 / 11 / 1023: 47$ & 59.6 & 16.5 & -28.1 & 35.0 & 51.2 & 19.0 \\
\hline & 12/11/10 1:07 & 54.1 & 15.0 & -27.8 & 36.0 & 53.4 & 19.4 \\
\hline & $12 / 11 / 101: 47$ & 51.3 & 15.7 & -27.8 & 37.4 & 54.6 & 20.0 \\
\hline & 12/11/10 18:00 & 38.7 & 10.3 & n.d. & 37.8 & 62.4 & 18.1 \\
\hline \multirow[t]{26}{*}{ Event 2} & $3 / 12 / 1018: 00$ & 41.7 & 3.5 & -28.1 & 32.9 & 77.4 & 7.4 \\
\hline & 4/12/10 5:49 & 82.8 & 7.4 & -28.9 & 28.1 & 54.5 & 8.0 \\
\hline & 4/12/10 6:29 & 120.9 & 7.7 & -29.0 & 28.5 & 55.0 & 8.0 \\
\hline & 4/12/10 7:09 & 150.2 & 9.4 & -28.9 & 27.8 & 49.7 & 8.2 \\
\hline & 4/12/10 7:49 & 173.9 & 10.8 & n.d. & n.d. & n.d. & n.d. \\
\hline & 4/12/10 8:29 & 177.3 & 11.6 & -28.8 & 26.7 & 41.8 & 8.8 \\
\hline & 4/12/10 9:09 & 177.3 & 12.2 & n.d. & 26.6 & 41.6 & 8.9 \\
\hline & 4/12/10 9:49 & 176.9 & 12.9 & -28.8 & 27.0 & 42.2 & 9.1 \\
\hline & 4/12/10 10:29 & 171.3 & 12.0 & -28.5 & 26.6 & 42.1 & 9.1 \\
\hline & 4/12/10 11:09 & 164.4 & 11.5 & -28.5 & n.d. & n.d. & n.d. \\
\hline & 4/12/10 11:49 & 162.4 & 11.2 & -28.3 & 26.6 & 44.2 & 9.2 \\
\hline & 4/12/10 12:29 & 161.2 & 11.1 & n.d. & 27.1 & 45.9 & 9.5 \\
\hline & 4/12/10 13:09 & 161.2 & 10.9 & -27.2 & 27.0 & 47.0 & 9.5 \\
\hline & 4/12/10 13:49 & 161.2 & 10.5 & -27.1 & 26.7 & 47.5 & 9.5 \\
\hline & $4 / 12 / 1014: 29$ & 148.1 & 10.2 & -27.1 & 26.8 & 48.0 & 9.6 \\
\hline & 4/12/10 15:09 & 145.8 & 10.1 & -27.2 & 27.0 & 49.4 & 9.8 \\
\hline & 4/12/10 15:49 & 133.1 & 10.3 & -27.2 & 27.2 & 50.2 & 10.0 \\
\hline & $4 / 12 / 10 \quad 16: 29$ & 127.4 & 9.8 & n.d. & 27.7 & 51.4 & 10.0 \\
\hline & 4/12/10 17:09 & 128.6 & 10.2 & -27.1 & 27.7 & 51.8 & 10.0 \\
\hline & 4/12/10 17:49 & 132.0 & 9.1 & -27.0 & 26.6 & 50.0 & 9.5 \\
\hline & $4 / 12 / 1018: 29$ & 148.2 & 11.1 & -27.0 & 26.8 & 49.6 & 9.7 \\
\hline & 4/12/10 19:09 & 154.1 & 9.9 & -26.9 & 26.5 & 49.1 & 9.7 \\
\hline & 4/12/10 19:49 & 154.1 & 10.2 & -26.9 & 27.3 & 50.4 & 9.8 \\
\hline & $4 / 12 / 1020: 29$ & 152.9 & 10.1 & -27.0 & 27.0 & 49.7 & 9.8 \\
\hline & 4/12/10 21:09 & 144.6 & 10.0 & n.d. & 27.0 & 50.1 & 9.9 \\
\hline & 5/12/10 18:00 & 98.5 & 7.4 & n.d. & 30.0 & 63.1 & 10.0 \\
\hline \multirow[t]{6}{*}{ Event 3} & 18/12/10 18:00 & 75.3 & 8.1 & n.d. & 30.3 & 76.6 & 5.1 \\
\hline & $19 / 12 / 106: 47$ & 88.8 & 6.6 & -28.9 & 27.8 & 54.2 & 7.8 \\
\hline & 19/12/10 7:27 & 187.4 & 6.7 & -28.4 & 25.4 & 49.9 & 7.6 \\
\hline & 19/12/10 8:07 & 244.8 & 8.5 & -28.6 & 23.4 & 41.8 & 7.1 \\
\hline & 19/12/10 8:47 & 307.6 & 10.7 & -28.6 & 25.6 & 37.7 & 7.2 \\
\hline & 19/12/10 9:27 & 379.1 & 11.1 & -28.7 & 21.3 & 31.8 & 6.7 \\
\hline
\end{tabular}




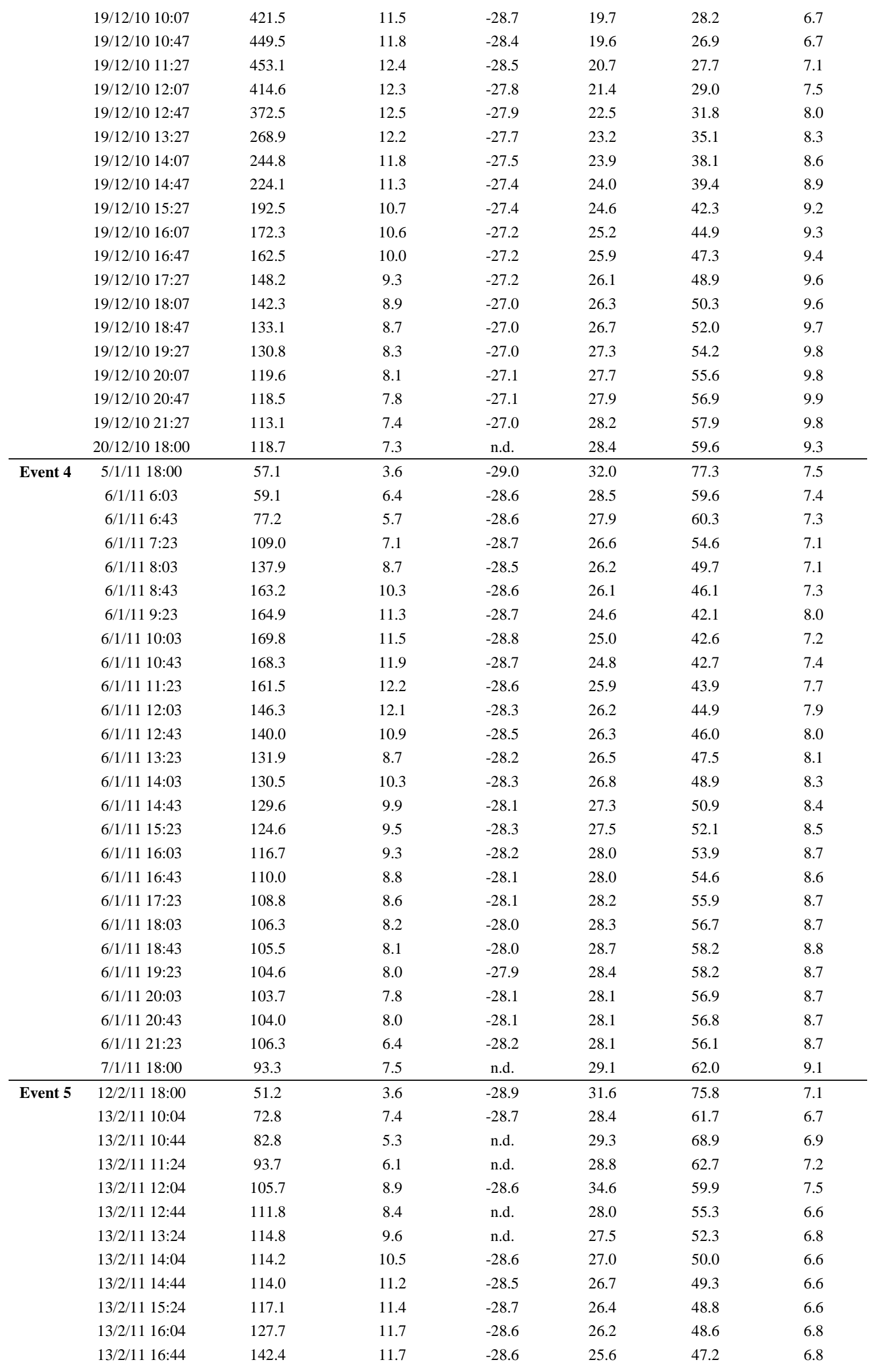




\begin{tabular}{|c|c|c|c|c|c|c|c|}
\hline & $13 / 2 / 11 \quad 17: 24$ & 159.0 & 11.9 & -28.5 & 25.2 & 45.9 & 6.7 \\
\hline & 13/2/11 18:04 & 167.3 & 12.8 & -28.6 & 25.0 & 44.5 & 6.8 \\
\hline & $13 / 2 / 1118: 44$ & 164.6 & 12.9 & -28.4 & 24.1 & 42.4 & 6.8 \\
\hline & $13 / 2 / 11 \quad 19: 24$ & 154.1 & 13.3 & -28.6 & 24.1 & 42.5 & 7.0 \\
\hline & 13/2/11 20:04 & 143.1 & 13.9 & -28.8 & 24.6 & 43.5 & 7.5 \\
\hline & $13 / 2 / 1120: 44$ & 132.2 & 14.1 & -28.5 & 25.6 & 44.9 & 7.5 \\
\hline & $13 / 2 / 1121: 24$ & 122.9 & 14.1 & -28.5 & 26.8 & 46.6 & 7.7 \\
\hline & $13 / 2 / 1122: 04$ & 114.6 & 13.8 & -28.6 & 27.5 & 48.8 & 8.0 \\
\hline & $13 / 2 / 1122: 44$ & 108.8 & 12.7 & -28.4 & 27.8 & 50.4 & 8.1 \\
\hline & $13 / 2 / 1123: 24$ & 104.1 & 12.2 & -28.5 & 28.0 & 52.1 & 8.1 \\
\hline & 14/2/11 0:04 & 100.3 & 11.4 & -28.5 & 28.6 & 54.4 & 8.3 \\
\hline & 14/2/11 0:44 & 96.2 & 11.0 & -28.5 & 28.8 & 56.9 & 6.7 \\
\hline & 14/2/11 1:24 & 93.1 & 10.2 & -28.7 & 28.9 & 57.0 & 8.6 \\
\hline & 14/2/11 18:00 & 79.5 & 6.6 & n.d. & 31.0 & 64.7 & 8.2 \\
\hline \multirow[t]{26}{*}{ Event 6} & 18/2/11 18:00 & 51.2 & 3.4 & -28.9 & 31.0 & 75.9 & 7.9 \\
\hline & $19 / 2 / 110: 31$ & 77.7 & 8.0 & -28.8 & 29.6 & 58.2 & 7.2 \\
\hline & 19/2/11 1:11 & 83.1 & 7.6 & -28.7 & 29.3 & 59.6 & 7.2 \\
\hline & 19/2/11 1:51 & 105.8 & 9.2 & -28.8 & 27.0 & 51.3 & 6.8 \\
\hline & $19 / 2 / 112: 31$ & 164.7 & 10.5 & -28.8 & 23.8 & 42.4 & 5.9 \\
\hline & 19/2/11 3:11 & 226.8 & 12.6 & -28.8 & 23.2 & 37.5 & 5.9 \\
\hline & $19 / 2 / 113: 51$ & 245.1 & 15.5 & -28.8 & 24.5 & 34.5 & 6.1 \\
\hline & 19/2/11 4:31 & 243.7 & 17.5 & -28.4 & 25.7 & 33.5 & 6.4 \\
\hline & 19/2/11 5:11 & 227.2 & 17.8 & -28.4 & 26.8 & 35.1 & 6.7 \\
\hline & $19 / 2 / 115: 51$ & 210.3 & 18.2 & -28.3 & 27.5 & 37.8 & 7.1 \\
\hline & 19/2/11 6:31 & 191.5 & 17.0 & -28.2 & 27.4 & 39.8 & 7.4 \\
\hline & 19/2/11 7:11 & 174.6 & 15.5 & -28.3 & 28.2 & 44.3 & 7.9 \\
\hline & 19/2/11 7:51 & 160.6 & 16.7 & -28.4 & 27.7 & 42.1 & 7.7 \\
\hline & 19/2/11 8:31 & 149.1 & 14.5 & -28.3 & 28.0 & 46.1 & 8.1 \\
\hline & 19/2/11 9:11 & 139.1 & 13.3 & -28.3 & 27.7 & 46.9 & 8.0 \\
\hline & 19/2/11 9:51 & 130.7 & 12.7 & -28.3 & 27.6 & 49.1 & 8.1 \\
\hline & 19/2/11 10:31 & 124.3 & 11.8 & -28.1 & 27.9 & 50.5 & 8.2 \\
\hline & $19 / 2 / 1111: 11$ & 119.3 & 11.3 & n.d. & 27.8 & 51.6 & 8.3 \\
\hline & $19 / 2 / 1111: 51$ & 115.2 & 10.5 & -28.1 & 27.4 & 51.9 & 8.2 \\
\hline & $19 / 2 / 11 \quad 12: 31$ & 111.0 & 10.4 & -28.3 & 28.4 & 54.8 & 8.5 \\
\hline & 19/2/11 13:11 & 107.8 & 10.1 & -28.3 & 28.0 & 55.2 & 8.6 \\
\hline & 19/2/11 13:51 & 104.6 & 9.0 & n.d. & 28.3 & 57.1 & 8.6 \\
\hline & $19 / 2 / 1114: 31$ & 102.5 & 9.1 & -28.2 & 27.9 & 55.7 & 8.5 \\
\hline & 19/2/11 15:11 & 99.5 & 8.6 & -28.2 & 28.4 & 58.4 & 8.6 \\
\hline & 19/2/11 15:51 & 98.4 & 8.0 & n.d. & 28.1 & 58.0 & 8.6 \\
\hline & 20/2/11 18:00 & 76.5 & 4.8 & n.d. & 30.8 & 72.1 & 8.8 \\
\hline
\end{tabular}

12 


\begin{tabular}{|c|c|c|c|c|}
\hline Site & Distance to stream (m) & SOC & WEOC* & DOC \\
\hline & & $\begin{array}{l}\delta^{13} \mathrm{C} \\
(\% o)\end{array}$ & $\begin{array}{l}\delta^{13} \mathrm{C} \\
(\% o)\end{array}$ & $\delta^{13} \mathrm{C}(\% \circ)$ \\
\hline Wetland domain & 10 & -29.8 & -28.6 & $\begin{array}{c}- \\
29.1 \pm 0.1 * *\end{array}$ \\
\hline Upland domain (PK2) & 95 & -27.0 & -25.6 & n.d. \\
\hline Upland domain (PK3) & 190 & -26.4 & -24.9 & n.d. \\
\hline Upland domain (PK4) & 240 & -26.5 & -24.3 & n.d. \\
\hline
\end{tabular}

* WEOC : Water Extractable Organic Carbon

** Data measured in riparian soils at the beginning of the hydrological period $\mathrm{B}$ before the inputs of ${ }^{13} \mathrm{C}$-enriched DOC from upland soils.

End of supplementary Table 2 


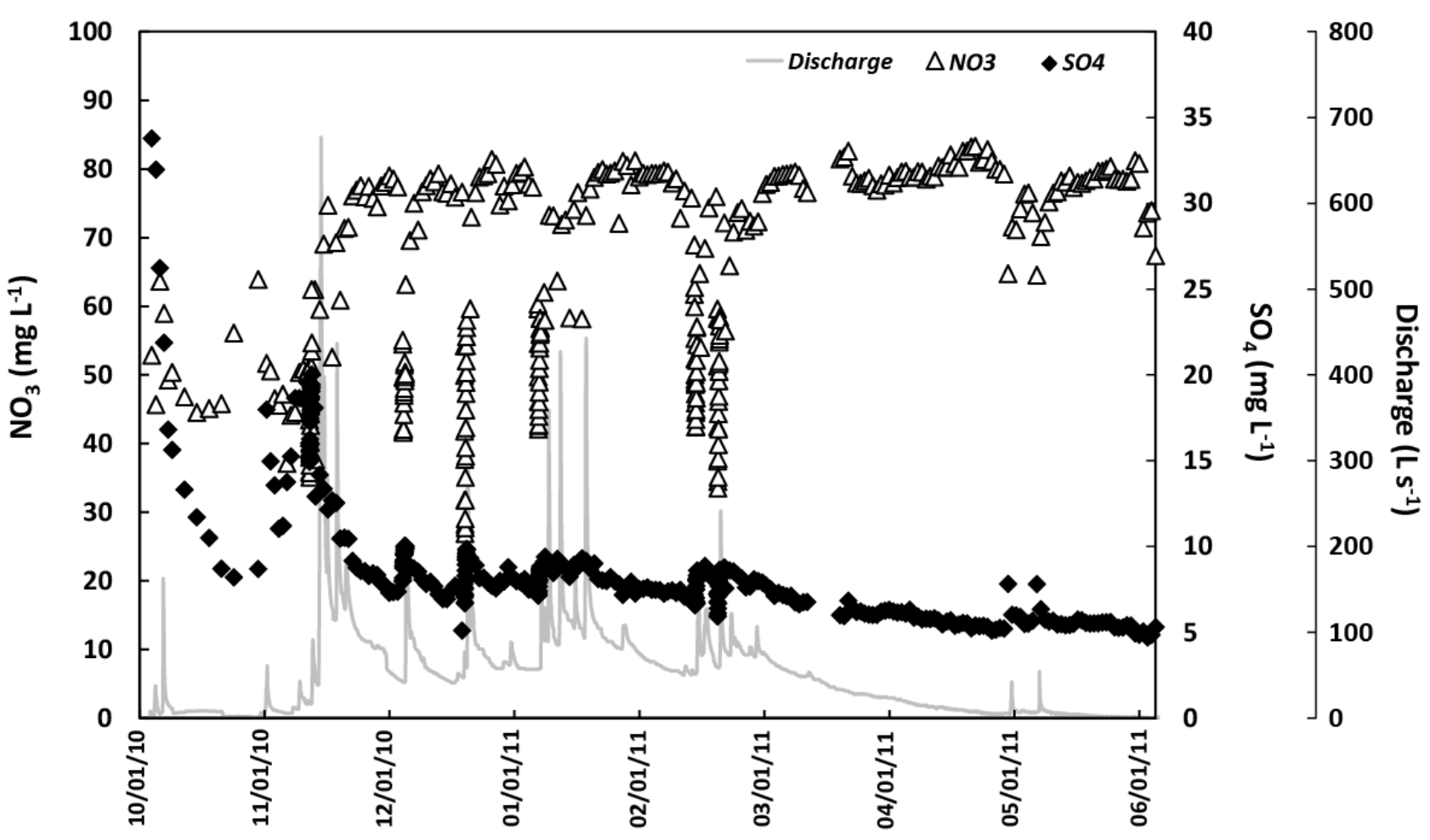

